\title{
OSCILLATIONS IN NEUTRAL EQUATIONS WITH PERIODIC COEFFICIENTS
}

\author{
G. LADAS, CH. G. PHILOS, AND Y. G. SFICAS
}

(Communicated by Kenneth R. Meyer)

ABSTRACT. We obtain a necessary and sufficient condition for the oscillation of all solutions of the neutral delay differential equation:

$$
\frac{d}{d t}[x(t)+p x(t-\tau)]+Q(t) x(t-\sigma)=0,
$$

where $p \in \mathbf{R}, Q \in C\left[[0, \infty), \mathbf{R}^{+}\right], Q$ is $\omega$-periodic with $\omega>0, Q(t) \not \equiv 0$ for $t \geqq 0$, and there exist positive integers $n_{1}$ and $n_{2}$ such that $\tau=n_{1} \omega$ and $\sigma=n_{2} \omega$. More precisely we show that every solution of (1) oscillates if and only if every solution of an associated neutral equation with constant coefficients oscillates.

\section{INTRODUCTION AND PRELIMINARIES}

Consider the neutral delay differential equation

$$
\frac{d}{d t}[x(t)+p x(t-\tau)]+Q(t) x(t-\sigma)=0,
$$

where

$$
p \in \mathbf{R}, Q \in C\left[[0, \infty), \mathbf{R}^{+}\right], Q \text { is } \omega \text {-periodic with } \omega>0,
$$
$Q(t) \not \equiv 0$ for $t \geqq 0$, and there exist positive integers $n_{1}$ and $n_{2}$ such that $\tau=n_{1} \omega$ and $\sigma=n_{2} \omega$.

Our aim in this paper is to obtain a necessary and sufficient condition for the oscillation of all solutions of (1). More precisely we will establish the following result.

Theorem 1. Assume that (2) is satisfied and set

$$
\tau_{1}=\int_{0}^{\tau} Q(s) d s \text { and } \sigma_{1}=\int_{0}^{\sigma} Q(s) d s .
$$

Then the following statements are equivalent.

(a) Every solution of (1) oscillates.

(b) Every solution of the neutral equation with constant coefficients

$$
\frac{d}{d t}\left[y(t)+p y\left(t-\tau_{1}\right)\right]+y\left(t-\sigma_{1}\right)=0
$$

oscillates.

Received by the editors March 9, 1990.

1980 Mathematics Subject Classification (1985 Revision). Primary 34K15; Secondary 34C10. 
This theorem shows that the oscillatory behavior of (1) when $Q$ is periodic and (2) holds is characterized by the oscillatory behavior of the linear autonomous neutral equation (4). On the other hand it is also known (see [12]) that every solution of (4) oscillates if and only if its characteristic equation

$$
\lambda+p \lambda e^{-\lambda \tau_{1}}+e^{-\lambda \sigma_{1}}=0
$$

has no real roots.

The way that we were led to Theorem 1 was by looking for a solution of (1) in the form

$$
x(t)=e^{\lambda \int_{0}^{t} Q(s) d s} .
$$

By substituting (6) into (1) we find

$$
e^{\lambda \int_{0}^{t} Q(s) d s}\left[\lambda Q(t)+p \lambda Q(t-\tau) e^{-\lambda \int_{t-\tau}^{t} Q(s) d s}+Q(t) e^{-\lambda \int_{t-\sigma}^{t} Q(s) d s}\right]=0 .
$$

Therefore if $Q$ is not identically zero and if $Q$ is $\omega$-periodic with $\tau$ and $\sigma$ integral multiples of $\omega$, then (7) reduces to (5) where $\tau_{1}$ and $\sigma_{1}$ are defined by (3). The fact that (5) characterizes the oscillatory behavior of (1) is a remarkable result that is not obvious.

The oscillatory behavior of neutral differential equations has been the subject of many recent investigations. See, for example [1]-[5], [8], and [12] and the references cited therein. The technique that we employ in the proof of Theorem 1 was initiated in [9] and [10] and had been used successfully in linear neutral autonomous equations. See, for example, [4], [5], and [13]. It is, however, surprising that the same technique may be modified to also apply to equations with periodic coefficients.

Let $\gamma=\max [\tau, \sigma\}$. By a solution of (1) we mean a function $x$ where $x \in$ $C\left[\left[t_{0}-\gamma, \infty\right), \mathbf{R}\right]$, for some $t_{0} \geqq 0$, such that $x(t)+p x(t-\tau)$ is continuously differentiable on $\left[t_{0}, \infty\right)$ and (1) is satisfied for $t \geqq t_{0}$.

Assume that $p \in \mathbf{R}, \tau, \sigma \in(0, \infty)$, and $Q \in C[[0, \infty), \mathbf{R}]$. Let $t_{0} \geqq 0$ be a given initial point and let $\phi \in C\left[\left[t_{0}-\gamma, t_{0}\right], \mathbf{R}\right]$ be a given initial function. Then by the method of steps, one can see that (1) has a unique solution $x \in$ $C\left[\left[t_{0}-\gamma, \infty\right), \mathbf{R}\right]$ such that

$$
x(t)=\phi(t) \text { for } t_{0}-\gamma \leqq t \leqq t_{0} .
$$

As is customary, a solution of (1) is said to oscillate if it has arbitrarily large zeros. Otherwise the solution is called nonoscillatory.

In the sequel, unless otherwise specified, when we write a functional inequality we will assume that it holds for all sufficiently large values of $t$.

\section{THE PROOF OF THEOREM 1}

When $p=0$, the proof of Theorem 1 is a consequence of known results. Indeed, in this case (1) reduces to

$$
\dot{x}(t)+Q(t) x(t-\sigma)=0,
$$


where

$$
Q \in C\left[[0, \infty], \mathbf{R}^{+}\right] \text {and } \sigma>0 .
$$

Now it is well known, see [6] and [7], that every solution of $\left(1^{\prime}\right)$ oscillates provided that

$$
\lim _{t \rightarrow \infty} \inf \int_{t-\sigma}^{t} Q(s) d s>\frac{1}{e} .
$$

It is also known (see [11]) that $\left(1^{\prime}\right)$ has a nonoscillatory solution provided that

$$
\sup _{t \geqq \sigma} \int_{t-\sigma}^{t} Q(s) d s \leqq \frac{1}{e} \text {. }
$$

Hence if $Q$ is $\sigma$-periodic and if we set

$$
\sigma_{1}=\int_{0}^{\sigma} Q(s) d s
$$

then every solution of $\left(1^{\prime}\right)$ oscillates if and only if

$$
\sigma_{1}>\frac{1}{e}
$$

that is, if and only if every solution of

$$
\dot{y}(t)+y\left(t-\sigma_{1}\right)=0
$$

oscillates (see [9]).

In view of the above discussion, in the sequel, we will assume that

$$
p \neq 0 \text {. }
$$

For the proof of $(a) \Rightarrow(b)$ assume, for the sake of contradiction, that (4) has a nonoscillatory solution. Then (5) must have a real root $\lambda_{0}$ (see [12]). Now by direct substitution into (1), one can see that

$$
x(t)=e^{\lambda_{0} \int_{0}^{t} Q(s) d s} \quad \text { for } t \geqq \max \{\tau, \sigma\}
$$

is a nonoscillatory solution of (1). This contradicts the hypothesis that every solution of (1) oscillates and completes the proof that $(a) \Rightarrow(b)$.

The proof that $(b) \Rightarrow(a)$ is quite involved and will be accomplished by establishing a series of lemmas.

In the sequel we will assume that (b) holds and that, for the sake of contradiction, (1) has an eventually positive solution that we will denote by $x(t)$. The proof will be completed when we reach a contradiction.

Set

$$
F(\lambda)=\lambda+p \lambda e^{-\lambda \tau_{1}}+e^{-\lambda \sigma_{1}} .
$$

The hypothesis that (b) holds is equivalent to the fact that (5) has no real roots (see [12]). As $F(0)=1$, it follows that

$$
F(\lambda)>0 \text { for } \lambda \in \mathbf{R} \text {. }
$$

Also $F(\infty)=\infty$ and clearly $F(-\infty)$ must be $\infty$, for, otherwise, $F(-\infty)=-\infty$ and so (5) would have a real root.

The following lemma is now an elementary consequence of the above observations. 
Lemma 1. The following statements are true.

(a) There exists a positive number $m_{0}$ such that

$$
\lambda+p \lambda e^{-\lambda \tau_{1}}+e^{-\lambda \sigma_{1}} \geqq m_{0} \text { for } \lambda \in \mathbf{R}
$$

or equivalently

$$
\lambda+p \lambda e^{\lambda \tau_{1}}-e^{\lambda \sigma_{1}} \leqq-m_{0} \text { for } \lambda \in \mathbf{R} .
$$

(b) If $p>0$ then $\sigma_{1}>\tau_{1}$ and $\sigma>\tau$.

The following lemma can easily be proved by direct substitution into (1) and its proof will be omitted.

Lemma 2. If $v(t)$ is a solution of (1) for $t \geqq t_{0} \geqq 0$ then

$$
w_{1}(t)=v(t)+p v(t-\tau) \quad \text { for } t \geqq t_{0}+\tau
$$

and

$$
w_{2}(t)=\int_{t-\sigma}^{t-\tau} Q(s) v(s) \quad \text { for } t \geqq t_{0}+\max \{\tau, \sigma\}
$$

are also solutions of (1).

Before we state the next lemma we introduce some notation.

Let $W^{0}$ be the set of all continuously differentiable solutions $w(t)$ of (1) with the properties that

$$
w(t)>0 \text { and } \dot{w}(t) \leqq 0 \text { for all large } t \text { and } \lim _{t \rightarrow \infty} w(t)=0 .
$$

Also let $W^{\infty}$ be that set of all continuously differentiable solutions $w(t)$ of (1) such that

$$
w(t)>0 \text { and } \dot{w}(t) \geqq 0 \quad \text { for all large } t \text { and } \lim _{t \rightarrow \infty} w(t)=\infty .
$$

For each $w \in W^{\infty}$ we define the set

$$
\wedge^{0}(w)=\left\{\lambda \in \mathbf{R}^{+}: \dot{w}(t)+\lambda Q(t) w(t) \leqq 0 \text { for all large } t\right\},
$$

and for each $w \in W^{0}$ we define the set

$$
\wedge^{\infty}(w)=\left\{\lambda \in \mathbf{R}^{+}:-\dot{w}(t)+\lambda Q(t) w(t) \leqq 0 \text { for all large } t\right\} .
$$

Clearly $0 \in \wedge^{0}(w)$ for every $w \in W^{0}$ and $0 \in \wedge^{\infty}(w)$ for every $w \in W^{\infty}$. It is also easy to see that for any $w \in W^{0} \cup W^{\infty}, \wedge^{0}(w)$ and $\wedge^{\infty}(w)$ are subintervals of $\mathbf{R}^{+}$.

The next three lemmas describe some interesting facts about the sets $W^{0}$ and $W^{\infty}$ and the sets $\wedge^{0} w$ and $\wedge^{\infty}(w)$ with $w \in W^{0} \cup W^{\infty}$.

Lemma 3. $W^{0} \cup W^{\infty} \neq \varnothing$. That is, there exists a solution $w$ of (1) that satisfies either (8) or (9).

Proof. Set

$$
y(t)=x(t)+p x(t-\tau),
$$


where $x(t)$ is an eventually positive solution of (1). Then

$$
\dot{y}(t)=-Q(t) x(t-\sigma) .
$$

As $Q(t) \geqq 0$ and $Q(t) \neq 0$, it follows that $\dot{w}(t) \leqq 0$ and so either eventually $y(t)>0$ or eventually $y(t)<0$.

First assume that eventually $y(T)>0$. Then we claim that $y \in W^{0}$. Otherwise

$$
\lim _{t \rightarrow \infty} y(t)=l>0
$$

Set

$$
v(t)=y(t)+p y(t-\tau) .
$$

Then

$$
\dot{v}(t)=-Q(t) y(t-\sigma)
$$

and so by (10) we obtain

$$
\dot{v}(t) \leqq-\frac{l}{2} Q(t) .
$$

Clearly $\int_{0}^{\infty} Q(s) d s=\infty$ and so by integrating (11) from $t_{0}$ to $\infty$, with $t_{0}$ sufficiently large, we are led to a contradiction.

Next assume that eventually $y(t)<0$. Set

$$
z(t)=-y(t)
$$

and observe that

$$
\dot{z}(t)=Q(t) x(t-\sigma) .
$$

Hence $z(t)$ is eventually positive and increasing and so either

$$
\lim _{t \rightarrow \infty} z(t)=\infty
$$

or

$$
\lim _{t \rightarrow \infty} z(t)=L \in(0, \infty) .
$$

If (12) holds then $z \in W^{\infty}$. On the other hand, if (13) holds, set

$$
u(t)=-[z(t)+p z(t-\tau)]
$$

and observe that eventually

$$
\dot{u}(t)=Q(t) z(t-\sigma) \geqq(L / 2) Q(t) .
$$

From this it is easily seen that $u \in W^{\infty}$. The proof of Lemma 3 is complete.

When $W^{0} \neq \varnothing$, the next lemma shows how to construct a sequence of functions $\left(w_{n}\right)$ in $W^{0}$ and a positive number $\lambda^{*}$ such that

$$
\lambda^{*} \in \wedge^{0}\left(w_{n}\right) \text { for all } n \text {. }
$$

Also a similar construction is shown when $W^{\infty} \neq \varnothing$. 
Lemma 4. The following statements hold.

(a) Assume that $W^{0} \neq \varnothing$. Then

$$
p>-1 \text {. }
$$

Let $w_{0} \in W^{0}$ and set $w(t)=w_{0}(t)+p w_{0}(t-\tau)$. Then $w \in W^{0}$. Furthermore, $1 \in \wedge^{0}(w)$ if $-1<p<0$ and $1 /(1+p) \in \wedge^{0}(w)$ if $p>0$.

(b) Assume that $W^{\infty} \neq \varnothing$. Then

$$
p<-1 \text {. }
$$

Let $w_{\infty} \in W^{\infty}$ and set $w(t)=-\left[w_{\infty}(t)+p w_{\infty}(t-\tau)\right]$ if $\sigma \leqq \tau$ and

$$
v(t)=-\left[w_{\infty}(t)+p w_{\infty}(t-\tau)\right]+\int_{t-\sigma}^{t-\tau} Q(s) w_{\infty}(s) d s \quad \text { if } \sigma>\tau .
$$

Then $w, v \in W^{\infty}$. Furthermore, $-1 / p \in \wedge^{\infty}(w)$ if $\sigma \leqq \tau$ and $\frac{1}{-p+\sigma_{1}-\tau_{1}} \in$ $\wedge^{\infty}(v)$ if $\sigma>\tau$.

Proof. (a) We have

$$
\dot{w}(t)=-Q(t) w_{0}(t-\sigma) \leqq 0
$$

and $\dot{w}(t)$ is not identically zero for all large $t$. Also

$$
\lim _{t \rightarrow \infty} w(t)=\lim _{t \rightarrow \infty}\left[w_{0}(t)+p w_{0}(t-\tau)\right]=0 .
$$

Thus $w(t)$ decreases to zero which implies that eventually $w(t)>0$. Hence $w \in W^{0}$. Now observe that

$$
0<w(t)=w_{0}(t)+p w_{0}(t-\tau) \leqq(1+p) w_{0}(t-\tau)
$$

which implies that (14) holds.

First, assume that $-1<p \leqq 0$. Then

$$
w(t)=w_{0}(t)+p w_{0}(t-\tau)<w_{0}(t)
$$

and so

$$
\dot{w}(t)=-Q(t) w_{0}(t-\sigma) \leqq-Q(t) w(t-\sigma) \leqq-Q(t) w(t)
$$

which shows that $1 \in \wedge^{0}(w)$.

Next, assume that $p>0$. By Lemma 1(b) we also have $\sigma>\tau$. Therefore,

$$
w(t)=w_{0}(t)+p w_{0}(t-\tau) \leqq(1+p) w_{0}(t-\tau) \leqq(1+p) w_{0}(t-\sigma)
$$

and so

$$
\dot{w}(t)=-Q(t) w_{0}(t-\sigma) \leqq-Q(t) w(t) /(1+p)
$$

which shows that

$$
1 /(1+p) \in \wedge^{0}(w) .
$$

(b) Set $z(t)=-\left[w_{\infty}(t)+p w_{\infty}(t-\tau)\right]$. Then

$$
z(t)=Q(t) w_{\infty}(t-\sigma)
$$


and one can easily see that $z \in W^{\infty}$. Hence

$$
0<z(t)=-\left[w_{\infty}(t)+p w_{\infty}(t-\tau)\right] \leqq-(1+p) w_{\infty}(t-\tau)
$$

which shows that (15) holds.

First assume that $\sigma \leqq \tau$. Then $w=z$ and so $w \in W^{\infty}$. Furthermore,

$$
-p w_{\infty}(t-\sigma) \geqq-p w_{\infty}(t-\tau)>w(t)
$$

and so

$$
0=-\dot{w}(t)+Q(t) w_{\infty}(t-\sigma) \geqq-\dot{w}(t)+Q(t) w(t) /-p
$$

which shows that

$$
-1 / p \in \wedge^{\infty}(w)
$$

Next assume that $\sigma>\tau$. Then from (16) we see that

$$
\dot{v}(t)=Q(t) w_{\infty}(t-\tau)
$$

from which it follows that $v \in W^{\infty}$. From (16) and (2) we see that

(18) $v(t)<-p w_{\infty}(t-\tau)+w_{\infty}(t-\tau) \int_{t-\sigma}^{t-\tau} Q(s) d s=w_{\infty}(t-\tau)\left(-p+\sigma_{1}-\tau_{1}\right)$.

It follows from (17) and (18) that

$$
-\dot{v}(t)+\frac{1}{-p+\sigma_{1}-\tau_{1}} Q(t) v(t) \leqq 0
$$

which shows that

$$
\frac{1}{-p+\sigma_{1}-\tau_{1}} \in \wedge^{\infty}(v) .
$$

The proof of Lemma 4 is complete.

Lemma 5. (a) Assume that $W^{0} \neq \varnothing$. Then there exists a $\lambda^{*} \in(0, \infty)$ such that $\lambda^{*}$ is an upper bound of $\wedge^{0}(w)$ for every $w \in W^{0}$.

(b) Assume that $W^{\infty} \neq \varnothing$. Then there exists a $\lambda^{*} \in(0, \infty)$ such that $\lambda^{*}$ is an upper bound of $\wedge^{\infty}(w)$ for every $w \in W^{\infty}$.

Proof. (a) As we proved in Lemma 4(a), (see (14)), in this case $p>-1$.

Case 1. $-1<p<0$. Let $w \in W^{0}$. Then, clearly, $w(t)+p w(t-\tau)$ decreases to zero and so eventually

$$
w(t)>-p w(t-\tau)
$$

Let $\lambda \in \wedge^{0}(w)$ and set

$$
\phi_{\lambda}(t)=w(t) e^{\lambda \int_{0}^{t} Q(s) d s}
$$

Then eventually

$$
\dot{\phi}_{\lambda}(t) \leqq 0
$$

and so $\phi_{\lambda}(t)$ is eventually decreasing. Hence eventually,

$$
w(t-\tau) e^{\lambda \int_{0}^{t-\tau} Q(s) d s} \geqq w(t) e^{\lambda \int_{0}^{t} Q(s) d s}
$$


and in view of (3) and (19)

$$
w(t-\tau) \geqq w(t) e^{\lambda \int_{t-\tau}^{t} Q(s) d s}=w(t) e^{\lambda \tau_{1}}>-p e^{\lambda \tau_{1}} w(t-\tau) .
$$

Hence

$$
-p e^{\lambda \tau_{1}}<1
$$

which shows that

$$
\lambda^{*}=\frac{1}{\tau_{1}} \ln \left(-\frac{1}{p}\right)
$$

is an upper bound of $\wedge^{0}(w)$.

Case 2. $p>0$. By Lemma $1(\mathrm{~b})$ in this case $\sigma>\tau$. Set

$$
\theta=\sigma-\tau \text { and } k=\int_{t-\theta}^{t} Q(s) d s \quad \text { for } t \geqq \theta .
$$

Clearly $k>0$. Now for every $t \geqq \theta$ let $t^{*}=t^{*}(t)$ be a point in $(t-\theta, t)$ such that

$$
\int_{t-\theta}^{t^{*}} Q(s) d s=\int_{t^{*}}^{t} Q(s) d s=\frac{k}{2} .
$$

Set

$$
z(t)=w(t)+p w(t-\tau) .
$$

Then eventually $z(t)>0$ and

$$
\dot{z}(t)=-Q(t) w(t-\sigma) .
$$

By integrating both sides of this equation from $t^{*}$ to $t$, for $t$ sufficiently large, we obtain

$$
z(t)-z\left(t^{*}\right)=-\int_{t^{*}}^{t} Q(s) w(s-\sigma) d s \leqq-w(t-\sigma) \frac{k}{2}
$$

Thus

$$
(k / 2) w(t-\sigma) \leqq z\left(t^{*}\right)-z(t) \leqq z\left(t^{*}\right)=w\left(t^{*}\right)+p w\left(t^{*}-\tau\right) \leqq(1+p) w\left(t^{*}-\tau\right)
$$

and so

$$
w\left(t^{*}-\tau\right) \geqq \frac{k}{2(1+p)} w(t-\sigma) .
$$

Let $\lambda \in \Lambda^{0}(w)$. Now by using the fact that the function $\phi_{\lambda}(t)$ which we defined in (20) is decreasing and in view of (21) we find,

$$
\begin{aligned}
w(t-\sigma) \geqq w\left(t^{*}-\tau\right) e^{\lambda \int_{t-\sigma}^{t^{*}-\tau} Q(s) d s} & =w\left(t^{*}-\tau\right) e^{\lambda \int_{t-\theta}^{t^{*}} Q(s) d s} \\
& \geqq \frac{k}{2(1+p)} w(t-\sigma) e^{\lambda k / 2}
\end{aligned}
$$

Hence

$$
\frac{k}{2(1+p)} e^{\lambda k / 2} \leqq 1
$$


which shows that

$$
\lambda^{*}=\frac{2}{k} \ln \frac{2(1+p)}{k}
$$

is an upper bound of $\wedge^{0}(w)$. The proof of part (a) of Lemma 5 is complete.

(b) As we proved in Lemma 4(b), (see (15)), in this case $p<-1$. Let $w \in W^{\infty}$ and set

$$
z(t)=-[w(t)+p w(t-\tau)] .
$$

Then eventually $z(t)>0$ and so

$$
w(t)<-p w(t-\tau)
$$

Let $\lambda \in \wedge^{\infty}(w)$ and set

$$
\psi_{\lambda}(t)=w(t) e^{-\lambda \int_{0}^{t} Q(s) d s}
$$

Then eventually

$$
\dot{\psi}_{\lambda}(t) \geqq 0
$$

and so $\psi_{\lambda}(t)$ is eventually increasing. Hence eventually,

$$
w(t) \geqq e^{\lambda \int_{t-\tau}^{t} Q(s) d s} w(t-\tau) \geqq e^{\lambda \tau_{1}} w(t) /(-p) .
$$

Therefore

$$
e^{\lambda \tau_{1}} /(-p) \leqq 1
$$

which shows that

$$
\lambda^{*}=\ln (-p) / \tau_{1}
$$

is an upper bound of $\wedge^{\infty}(w)$. The proof of Lemma 5 is complete.

On the basis of the preceding lemmas it suffices to examine each of the following four cases and in each case it remains to obtain a contradiction.

Case I: $W^{0} \neq \varnothing$ and $-1<p<0$;

Case II: $W^{0} \neq \varnothing$ and $p>0$;

Case III: $W^{\infty} \neq \varnothing$ and $\sigma \leqq \tau$;

Case IV: $W^{\infty} \neq \varnothing$ and $\sigma>\tau$.

Our strategy in each case is to use Lemma 4 to construct a sequence of functions $\left\{w_{n}\right\}$ such that for each $n=1,2, \ldots$,

$$
w_{n} \in W^{0} \text { in Cases I and II }
$$

and

$$
w_{n} \in W^{\infty} \text { in Cases III and IV. }
$$

In each case we will also find a positive number $\mu$ and show that for every $n=1,2, \ldots$,

$$
\text { if } \lambda \in \wedge^{0}\left(w_{n}\right) \text { then } \lambda+\mu \in \wedge^{0}\left(w_{n+1}\right)
$$

and

$$
\text { if } \lambda \in \wedge^{\infty}\left(w_{n}\right) \text { then } \lambda+\mu \in \wedge^{\infty}\left(w_{m+1}\right)
$$


In view of Lemma 5, (23) and (24) will eventually lead to the desired contradiction.

Case I. $W^{0} \neq \varnothing$ and $-1<p<0$. Let $w_{0} \in W^{0}$. Then clearly each of the functions

$$
w_{n}(t)=w_{n-1}(t)+p w_{n-1}(t-\tau) \text { for } n=1,2, \ldots
$$

belongs to $W^{0}$. We will now show that (23) holds with $\mu=m_{0}$, where $m_{0}$ is the constant in Lemma $1(\mathrm{a})$. To this end, let $\lambda \in \wedge^{0}\left(w_{n}\right)$ and set

$$
\phi_{\lambda}(t)=w_{n}(t) e^{i \int_{0}^{t} Q(s) d s} .
$$

Then eventually $\dot{\phi}_{\lambda}(t) \leqq 0$ and so eventually $\phi_{\lambda}(t)$ is a decreasing function. Finally observe that

$$
\begin{gathered}
\dot{w}_{n+1}(t)+(\lambda+\mu) Q(t) w_{n+1}(t) \\
=-Q(t) w_{n}(t-\sigma)+(\lambda+\mu) Q(t) w_{n}(t)+(\lambda+\mu) Q(t) p w_{n}(t-\tau) \\
=Q(t)\left[-\phi_{\lambda}(t-\sigma) e^{-\lambda \int_{0}^{t-\sigma} Q(s) d s}+(\lambda+\mu) \phi_{\lambda}(t) e^{-\lambda \int_{0}^{t} Q(s) d s}\right. \\
+(\lambda+\mu) p \phi_{\lambda}(t-\tau) e^{\left.-\lambda \int_{0}^{t-\tau} Q(s) d s\right]} \\
\leqq Q(t) \phi_{\lambda}(t) e^{-\lambda \int_{0}^{t} Q(s) d s}\left(-e^{\lambda \sigma_{1}}+\lambda+\mu+\lambda p e^{\lambda \tau_{1}}+\mu p e^{\lambda \tau_{1}}\right) .
\end{gathered}
$$

By using Lemma 1(a) and the fact that $p<0$ we see that

$$
\begin{aligned}
\dot{w}_{n+1}(t)+\left(\lambda+m_{0}\right) Q(t) w_{n+1}(t) & \leqq Q(t) \phi_{\lambda}(t) e^{-\lambda \int_{0}^{t} Q(s) d s}\left(-m_{0}+m_{0}\right) \\
& =0
\end{aligned}
$$

This shows that $\lambda+m_{0} \in \wedge^{0}\left(w_{n+1}\right)$ and the proof in Case $I$ is complete.

Case II. $W^{0} \neq \varnothing$ and $p>0$. By Lemma $1(\mathrm{~b})$ we know that $\sigma>\tau$. Here we will also use the sequence (25). Finally we claim that (23) holds with

$$
\mu=m_{0} / 1+p e^{\lambda^{*} \tau_{1}}
$$

where $\lambda^{*}$ is the constant in Lemma 5(a). Indeed (26) implies that

$$
\begin{aligned}
& \dot{w}_{n+1}(t)+(\lambda+\mu) Q(t) w_{n+1}(t) \\
& \quad \leqq Q(t) \phi_{\lambda}(t-\tau) e^{-\lambda \int_{0}^{t} Q(s) d s}\left(-e^{\lambda \sigma_{1}}+\lambda+\mu+\lambda p e^{\lambda \tau_{1}}+\mu p e^{\lambda \tau_{1}}\right) \\
& \quad \leqq Q(t) \phi_{\lambda}(t-\tau) e^{-\lambda \int_{0}^{t} Q(s) d s}\left[-m_{0}+\mu\left(1+p e^{\lambda \tau_{1}}\right)\right] \\
& \quad \leqq Q(t) \phi_{\lambda}(t-\tau) e^{-\lambda \int_{0}^{t} Q(s) d s}\left[-m_{0}+\mu\left(1+p e^{\lambda^{*} \tau_{1}}\right)\right] \\
& \quad=0 .
\end{aligned}
$$

This shows that $\lambda+\mu \in \wedge^{0}\left(w_{n+1}\right)$ and the proof in Case II is complete. 
Case III. $W^{\infty} \neq \varnothing$ and $\sigma \leqq \tau$. In this case we arrive at a contradiction by using the sequence

$$
w_{n}(t)=-\left[w_{n-1}(t)+p w_{n-1}(t-\tau)\right] \text { for } n=1,2, \ldots,
$$

where $w_{0}$ is some fixed element of $W^{\infty}$ and by taking

$$
\mu=m_{0} /(-p-1) \text {. }
$$

Of course, $p<-1$ in this case. The proof is as in Cases I and II but here we utilize the substitution

$$
\psi_{\lambda}(t)=e^{-\lambda \int_{0}^{t} Q(s) d s} w_{n}(t) .
$$

Case IV. $W^{\infty} \neq \varnothing$ and $\sigma>\tau$. In this case we use the sequence $w_{n}(t)=-\left[w_{n-1}(t)+p w_{n-1}(t-\tau)\right]+\int_{t-\sigma}^{t-\tau} Q(s) w_{n-1}(s) d s$ for $n=1,2, \ldots$, where $w_{0}$ is some fixed element of $W^{\infty}$. We also take

$$
\mu=\frac{m_{0}}{-p-1+\left(1 / \lambda_{0}\right)} \quad \text { with } \quad \lambda_{0}=\frac{1}{-p+\sigma_{1}-\tau_{1}}
$$

which by Lemma $4(\mathrm{~b})$ lies in $\wedge^{\infty}\left(w_{n}\right)$ for every $n=1,2, \ldots$ In this case we also use (27) with $\lambda \geqq \lambda_{0}$.

The proof of Theorem 1 is complete.

\section{REFERENCES}

1. Q. Chuanxi and G. Ladas, Oscillations of neutral differential equations with variable coeffcients, Applicable Anal. (to appear).

2. M. K. Grammatikopoulos, E. A. Grove, and G. Ladas, Oscillations of first-order neutral delay differential equations, J. Math. Anal. Appl. 120 (1986), 510-520.

3. M. K. Grammatikopoulos, G. Ladas, and Y. G. Sficas, Oscillation and asymptotic behavior of neutral equations with variable coefficients, Radovi Matematicki 2 (1986), 279-303.

4. M. K. Grammatikopoulos, Y. G. Sficas, and I. P. Stavroulakis, Necessary and sufficient conditions for oscillation of neutral equations with several coefficients, J. Differential Equations 76 (1988), 294-311.

5. E. A. Grove, G. Ladas, and A. Meimaridou, A necessary and sufficient condition for the oscillation of neutral equations, J. Math. Anal. Appl. 126 (1987), 241-354.

6. R. G. Koplatadze and T. A. Canturija, On the oscillatory and monotone solutions of the first order differential equations with deviating arguments, Defferential' nye Uravnenija 18 (1982), 1463-1465.

7. G. Ladas, Sharp conditions for oscillations caused by delays, Applicable Anal. 9 (1979), 95-98.

8. G. Ladas and Y. G. Sficas, Oscillations of neutral delay differential equations, Canad. Math. Bull. 29 (1986), 438-445.

9. G. Ladas, Y. G. Sficas, and I. P. Stavroulakis, Necessary and sufficient conditions for oscillations, Amer. Math. Monthly 90 (1983), 637-640.

10. _ Necessary and sufficient conditions for oscillations of higher order delay differential equations, Trans. Amer. Math. Soc. 285 (1984), 81-90.

11. Ch. G. Philos, On the existence of nonoscillatory solutions tending to zero at $\infty$ for differential equations with positive delays, Arch. Math. 36 (1980), 168-178. 
12. Y. G. Sficas and I. P. Stavroulakis, Necessary and sufficient conditions for oscillations of neutral differential equations, J. Math. Anal. Appl. 123 (1987), 494-507.

(G. Ladas) Department of MAthematics, The University of Rhode IsLand, Kingston, RHODE ISLAND 02881-0816

Department of Mathematics, University of Ioannina, P.O. Box 1186, Ioannina 45110, GREECE 